Espac. Saúde. 2021;22:e794 Doi 10.22421/1517-7130/es.2021v22.e794

\title{
Aspectos relacionados à implementação da Política Nacional de Atenção Integral à Saúde do Homem (PNAISH) no Brasil
}

\author{
Aspects related to the implementation of the Brazilian Policy for \\ Comprehensive Men's Health Care (PNAISH)
}

Aspectos relacionados con la implementación de la Política Nacional de Atención Integral de Salud del Hombre (PNAISH) en Brasil

Juliana de Pontes Nobre ${ }^{1}$, Consuelo Aires de Freitas ${ }^{2}$

1 Enfermeira. Mestre em Cuidados Clínicos em Enfermagem e Saúde. Universidade Estadual do Ceará, Fortaleza - Ceará

2 Enfermeira. Pós-doutora em Bioética e Ética Aplicada e Saúde Coletiva, Professora do Programa de Pós-Graduação Cuidados Clínicos em Enfermagem e Saúde da Universidade Estadual do Ceará,

Fortaleza - Ceará

\section{RESUMO}

Com a promulgação da CF de 1988, a saúde foi reconhecida como direito de todos e dever do estado. A saúde masculina deve ser regida de acordo com princípios e diretrizes do SUS. O propósito da PNAISH é garantir o cuidado ao homem, qualificando profissionais para atender esta população. O objetivo foi identificar produções bibliográficas sobre desafios na implementação da PNAISH na perspectiva da formação em enfermagem. Trata-se de revisão integrativa de periódicos indexados na LILACS, SCOPUS e BDENF acerca da temática entre

Autor de Correspondência:

*Juliana de Pontes Nobre. E-mail: jupnobre@hotmail.com 
2014 e 2019. A coleta de dados ocorreu entre novembro e dezembro de 2019. Procedeu-se a leitura do material, com identificação de três aspectos: implementação da PNAISH, a não adesão da população masculina à PNAISH, formação dos profissionais de enfermagem para saúde do homem. Concluiu-se que a saúde do homem está aquém de outros programas da saúde, é necessário investimento em formação. O tema carece de pesquisas aprofundadas.

Palavras-chave: Saúde do Homem. Cuidado de Enfermagem. Educação em Enfermagem.

\begin{abstract}
With the promulgation of the Federal Constitution of 1988, health was recognized as a right of all citizens and the State's responsibility. Men's health must be governed according to the principles and guidelines of the Brazilian Public Health System. The purpose of the Brazilian Policy for Men's Health care is to ensure care for men, qualifying professionals to serve this population. The objective was to identify papers on challenges in the implementation of the Brazilian Policy for Men's Health Care from the perspective of nursing education. This is an integrative review of journals indexed in LILACS, SCOPUS, and BDENF on the subject between 2014 and 2019. Data collection occurred between November and December 2019. The material was read, providing the identification of three aspects: implementation of the Policy, the nonadherence of the male population to the policy, training of nursing professionals for men's health. It was concluded that men's health is behind other health programs, with investment in training being required. The theme lacks in-depth research.
\end{abstract}

Keywords: Male health. Nursing care. Nursing education.

\title{
RESUMEN
}

Con la promulgación de la Constitución de 1988, la salud fue reconocida como derecho de todos y deber del Estado. La salud de los hombres debe regirse por los principios y directrices del SUS. La finalidad del PNAISH es asegurar la atención a los hombres y formar profesionales para atender esta población. El objetivo fue identificar producciones sobre desafíos en la implementación del PNAISH y la educación en enfermería. Revisión integradora de las revistas indexadas en LILACS, SCOPUS y BDENF entre 2014 y 2019. La recogida de datos se produjo entre noviembre y diciembre de 2019. Tras la lectura se identificaron tres aspectos: implantación del PNAISH, no adhesión de la población masculina al PNAISH y formación de enfermeros de salud masculina. Se concluyó que la salud de los hombres va a la zaga de otros programas de salud, por lo que es necesario invertir en capacitación. El tema necesita de más investigación.

Palabras clave: Salud masculina. Cuidado de enfermera. Educación en enfermería. 


\section{INTRODUÇÃO}

O processo de institucionalização do Sistema Único de Saúde (SUS), com posterior regulamentação pela Lei Orgânica da Saúde (LOS) nº 8.080/1990 e descentralização administrativo-financeira, fomentou avanços e mudanças por conta da necessidade de um modelo de atenção à saúde que garantisse acesso às ações e serviços para a população, com base nas diretrizes e princípios que estrategicamente estabeleceram uma episteme doutrinária e uma lógica organizativa. Tal processo influenciou fortemente o mercado de trabalho em saúde, caracterizado pela produção de serviços consumidos quando produzidos, com a subjetividade própria do cuidado prestado por cada trabalhador, que afeta e é afetado, com sentidos e sentimentos próprios $^{1}$.

Em 2009 o Ministério da Saúde lançou, como uma das prioridades de governo, a Política Nacional de Atenção Integral à Saúde do Homem (PNAISH), que tem como um dos de seus principais objetivos, promover ações de saúde que contribuam significativamente para a compreensão da realidade singular masculina nos seus diversos contextos socioculturais e político-econômicos ${ }^{2}$. Dentre os objetivos específicos descritos na PNAISH, a ação de formar e qualificar os profissionais da rede básica para o correto atendimento à saúde do homem provoca a inquietação sobre o que está sendo produzido no Brasil.

O processo de formação em enfermagem é dinâmico, deve estar em consonância direta com o setor da saúde, que é uma área que sofre constantes mudanças e avanços no conhecimento, através da pesquisa, introdução de novas tecnologias, etc. Por esta razão, é essencial que os profissionais de saúde, especialmente o enfermeiro, se atualizem e complementem sua formação acadêmica, tendo como objetivo oferecer assistência de qualidade e uma prática baseada em evidências científicas ${ }^{3}$.
Dentre as demandas contemporâneas na saúde, a extensão da cobertura do serviço à população masculina, mediante o conhecimento por parte dos profissionais e usuários e a implementação da PNAISH, surge como essencial à promoção da qualidade assistencial, considerando os desafios vivenciados pelos profissionais da saúde em sua prática diária, especialmente quando associada às causas de morbimortalidade no SUS, alicerce para o planejamento e a tomada de decisões dos gestores ${ }^{4}$.

Portanto, esse tema ainda tão preocupante no que se refere à incorporação nas práticas dos serviços de saúde, em que, nas experiências de docência e de serviço, observam-se lacun as e desigualdades na atenção à saúde do homem, elegeu-se ser pertinente um levantamento bibliográfico, tendo em vista que já se passaram mais de dez anos da promulgação da política, tempo suficiente para que produções científicas pudessem ser compartilhadas.

A relevância desse estudo consiste em buscar na literatura, conhecimento e experiências acerca da temática, levantando informações atualizadas e verificando se o conhecimento científico produzido a respeito do ensino da enfermagem para atenção integral à saúde do homem está sendo adequado de forma a contemplar as diretrizes e objetivos preestabelecidos na PNAISH, além de investigar se a demanda de saúde da população masculina está sendo suprida.

A partir da identificação do tema, definiu-se a questão norteadora do estudo: "O que vem sendo produzido sobre o processo de implementação e o ensino da PNAISH?” e o objetivo do estudo, que consistiu em identificar as produções bibliográficas sobre os desafios na implementação da PNAISH na perspectiva da formação em enfermagem, publicadas no período de 2014 a 2019, analisando o conhecimento produzido na temática. 


\section{MÉTODO}

Trata-se de uma revisão integrativa de periódicos de enfermagem indexados nas bases LILACS (Literatura Latino-americana e do Caribe em Saúde), BDENF (Base de Dados de Enfermagem) e SCOPUS, acerca da temática no período de 2014 a 2019. O estudo reuniu informações que possibilitam averiguar condições e ações do objeto para melhor planejar e proporcionar as práticas de saúde ${ }^{5}$.

Foram consideradas publicações que abordassem a temática Saúde do Homem, utilizando-se os seguintes descritores: Saúde do Homem, cuidado integral e ensino. A coleta de dados foi realizada nos meses de novembro e dezembro de 2019.

A pesquisa foi desenvolvida utilizando o operador boleano AND associando os descritores, o que possibilitou capturar 96 trabalhos. Depois de aplicados os critérios de inclusão (artigos completos acerca da temática, disponíveis on-line em português, publicados entre 2014 e 2019), foram excluídos livros, teses, dissertações, monografias, capítulos ou resenhas de livros, manuais, relatórios técnicocientíficos, artigos incompletos, não disponíveis online, em outros idiomas e que não tivessem relação com os descritores. A amostra constituiu-se assim de 10 artigos.

A análise dos dados foi pautada na literatura acerca do ensino de enfermagem na perspectiva da Política Nacional de Atenção Integral à Saúde do Homem (PNAISH), temáticas abordadas nos artigos pesquisados.

\section{RESULTADOS}

Para melhor compreensão, o Quadro 1 apresenta os artigos investigados segundo a referência do estudo, foco de atenção e caracterização do conteúdo.

Procedeu-se o exame do material que compreendeu leitura exaustiva, o que proporcionou a identificação de três categorias de abordagem que possibilitaram responder à questão norteadora: implementação da PNAISH, aspectos da não adesão da população masculina à PNAISH, formação e capacitação dos profissionais de enfermagem a saúde do homem.

O conteúdo apreendido acerca do foco da Implementação da PNAISH enfoca questões relacionadas à falhas nos processos de implementação da política como, por exemplo, ações centradas em aspectos clínicos assistenciais e/ou na saúde sexual e aparelho genital masculino, com foco apenas na prevenção de câncer de próstata; funcionamento das Unidades Básicas de Saúde (UBS) restrito ao horário comercial, impossibilitando o acesso do homem, normalmente tido como provedor da família e gerador de renda; falta de acolhimento adequado ao público masculino, na perspectiva preconizada pela PNAISH.

Quanto ao foco intitulado Aspectos da não adesão da população masculina ao serviço, pontuou-se como conteúdos manifestos as barreiras na procura do serviço pela população masculina, a falta de tempo, o medo e vergonha. Esses aspectos são muito comuns na sociedade. Não sendo distante da cultura masculina que ainda distancia o homem da procura na prevenção do câncer de próstata, em especial, ou até mesmo da procura do serviço de saúde por outro motivo. A falta de tempo não foi investigada a ponto de se entender como motivo relacionado aos aspectos anteriores. No entanto, sabe-se das dificuldades de usuários procurarem os serviços de saúde quando esses estão trabalhando ativamente. É uma realidade que não se pode desconsiderar.

Os aspectos falhos no acolhimento, fragilidade da informação/falta de conhecimento sobre o serviço e estratégias para captação da população masculina estão implicados numa relação que advém do processo de trabalho no serviço. Sabe-se que a gestão dos sujeitos trabalhadores perpassa por uma filosofia de entender a lógica do serviço. Os desdobramentos que 
acontecem junto aos usuários são reflexos da gestão. Então, o acolhimento é sistêmico em decorrência do trabalho do grupo e não isoladamente.

A formação e capacitação dos profissionais de enfermagem à Saúde do Homem é o foco central do presente trabalho, no qual se apreendeu nos estudos bibliográficos que há capacitação inadequada dos profissionais de enfermagem em serviço, bem como falha na formação dos profissionais de enfermagem. Tais conteúdos apreendidos nos referidos estudos deve-se a estratégias inadequadas no ensino em sala de aula acerca das Diretrizes da PNAISH, acontecendo um distanciamento entre conteúdos teóricos e as práticas no serviço. Ao passo que, quando se desenvolvem estratégias que favoreçam um alinhamento entre as práticas e saberes, fortalecese a formação dos enfermeiros.

Quadro 1 - Demonstrativo dos conteúdos encontrados nos artigos de enfermagem.

\begin{tabular}{|c|c|c|}
\hline AUTORES & FOCO DO CONTEÚDO & CATEGORIAS DE ANÁLISE \\
\hline $\begin{array}{l}\text { Rodrigues VME, Crispim CLM, } \\
\text { dos Santos RM, dos Anjos DS } \\
\text { Silveira CLG, Melo VFC, Barreto } \\
\text { AJR }^{7} \\
\text { Silva GA, Cunha LP, Freire MAM, } \\
\text { Tocantins FR }\end{array}$ & $\begin{array}{l}\text { Implementação da } \\
\text { PNAISH }\end{array}$ & $\begin{array}{l}\text { Falha no processo de implementação da } \\
\text { PNAISH. } \\
\text { Ações centradas em aspectos clínicos } \\
\text { e na saúde sexual e aparelho genital } \\
\text { masculino. Falta de acolhimento } \\
\text { adequado ao público masculino. } \\
\text { Escassez de estudos e publicações sobre } \\
\text { a PNAISH. } \\
\text { Falta de adesão dos homens, } \\
\text { principalmente pelo horário que o } \\
\text { serviço é oferecido. } \\
\text { Implementação de instrumentos que } \\
\text { viabilizem a implementação da PNAISH. }\end{array}$ \\
\hline $\begin{array}{l}\text { Barreto EF et al. }{ }^{9} \\
\text { Aguiar RS, Santana DC, Santana } \\
\text { PC }^{10} \\
\text { Alves BMS et al }{ }^{11}\end{array}$ & $\begin{array}{l}\text { Aspectos da não adesão } \\
\text { da população masculina } \\
\text { ao serviço. }\end{array}$ & $\begin{array}{l}\text { Barreiras na procura do serviço pela } \\
\text { população masculina (falta de tempo, } \\
\text { medo, vergonha). } \\
\text { Falha no acolhimento. } \\
\text { Feminização das UBS. } \\
\text { Fragilidade de informação/falta de } \\
\text { conhecimento sobre o serviço. } \\
\text { Estratégias de captação da população } \\
\text { masculina. }\end{array}$ \\
\hline $\begin{array}{l}\text { Ribeiro DB et al }{ }^{12} \\
\text { Sousa AP, Silva PES, Dias, RS, } \\
\text { Azevedo PR, Silva LDC }{ }^{13} \\
\text { Adamy EK, Lima TL, Teixeira DC, } \\
\text { Kutszepa BD, Bernardi GK }{ }^{14} \\
\text { Freitas ER et al }{ }^{15}\end{array}$ & $\begin{array}{l}\text { Formação e capacitação } \\
\text { dos profissionais de } \\
\text { enfermagem à saúde do } \\
\text { homem }\end{array}$ & $\begin{array}{l}\text { Capacitação inadequada dos } \\
\text { profissionais de enfermagem em serviço. } \\
\text { Falha na formação dos profissionais de } \\
\text { enfermagem. } \\
\text { Implantação das Diretrizes da } \\
\text { Política em sala de aula, ajudando os } \\
\text { enfermeiros e alunos no reconhecimento } \\
\text { e alinhamento das ações pautadas na } \\
\text { PNAISH. }\end{array}$ \\
\hline
\end{tabular}




\section{DISCUSSÃO}

Ressalta-se a escassez de estudos e publicações sobre o tema e a elaboração de instrumentos que viabilizem a implementação da PNAISH de forma sistemática e operacional em cada contexto de serviço. Percebese que a temática possui um processo construtivo lento e pouco explorado por parte dos serviços e de profissionais, visto o número reduzido de publicações científicas ${ }^{8}$. Este aspecto dificulta a incorporação de saberes nos diferentes contextos em que ocorrem a construção do conhecimento e as práticas de saúde. Entende-se que a atualização constante, a busca por conhecimento, além de mudanças nos serviços de saúde a fim de motivar a capacitação dos profissionais podem auxiliar a consolidação das políticas públicas brasileiras $^{14,15}$.

O aspecto apreendido como feminização da UBS está diretamente relacionado à cultura que é muito forte nas práticas e serviços com a priorização da atenção à mulher. Muito embora seja possível observar nas vivências cotidianas as transformações socioculturais e de gênero ocorridas no País, a saúde dos homens apresenta uma abordagem generalista, superficial, fragmentada e bastante descontextualizada do ambiente sociocultural dos homens, sendo necessário estruturar os serviços de saúde em termos de organização e processo do trabalho a fim de atender à especificidade dessa população ${ }^{11,12,13}$. Sabe-se que o exame retal é o mais próximo da detecção de alguma alteração na glândula prostática, contudo o medo, a vergonha de expor seus problemas, são empecilhos para que a população masculina não busque os cuidados preventivos necessários para a manutenção da sua saúde ${ }^{7}$, causando resistência na realização desse exame.

A PNAISH enfatiza a necessidade de mudanças de paradigmas no que concerne à percepção da população masculina em relação ao cuidado com a sua saúde e à saúde de sua família, no intuito de favorecer uma visão e postura crítico-reflexiva para que a política efetivamente aconteça em nosso País e o homem possa de maneira qualificada e humanizada ter acesso à saúde, um direito conquistado9.

A fragilidade da informação e a falta de conhecimento são consequências das falhas no acolhimento quando se compreende que este consiste numa relação entre usuário e trabalhador de saúde. Usuários, nessa perspectiva, devem ser participantes do processo. Observamos que embora os enfermeiros reconheçam as dificuldades enfrentadas por eles na assistência ao público masculino isso não tem sido suficiente para modificar a realidade de saúde dessa população na Estratégia Saúde da Família ${ }^{10}$. Tornase imprescindível o planejamento e a organização do serviço para atender a esta demanda com adoção de ações educativas que favoreçam a aderência da população masculina à atenção primária .

\section{CONCLUSÕES}

O presente estudo mostrou que a saúde do homem ainda está muito aquém de outros programas da atenção em saúde. Observa-se vários aspectos que dificultam o acesso da população masculina aos serviços de saúde, sendo que, dentre os mais importantes citados nos artigos estão a falta de acolhimento ou acolhimento inadequado, gerando receio no homem que procura a unidade de saúde, e o desconhecimento sobre as ofertas dos serviços das unidades de saúde. Pode-se considerar que os aspectos levantados pelos autores supracitados advêm de questões processuais no trabalho de ordem sistêmica, desde a implantação, implementação e avaliação.

A formação dos profissionais de saúde, sobretudo a do enfermeiro, no sentido de prepará-lo para desenvolver estratégias no atendimento das necessidades de saúde e desafios no SUS, ainda carece de investimento em educação permanente nos diversos níveis de atenção à saúde. 
Apesar de ser um tema de grande importância para a elaboração de políticas e ações no âmbito da saúde, as ações voltadas para o bem-estar do homem ainda necessitam de investigações e publicações aprofundadas.

\section{REFERÊNCIAS}

1. Ximenes Neto FRG, Lopes Neto D, Cunha ICKO, Ribeiro MA, Freire NP, Kalinowski CE, et al. Reflexões sobre a formação em Enfermagem no Brasil a partir da regulamentação do Sistema Único de Saúde. Cienc Saúde Coletiva [Internet]. 2020 [access at: July 02, 2020];25(1):3746.

2. Brasil. Ministério da Saúde (MS). Secretaria de Atenção à Saúde. Departamento de Ações Programáticas Estratégicas. Política Nacional de Atenção Integral à Saúde do Homem (princípios e diretrizes). Brasília: MS; 2008.

3. Ortega MC, Cecagno D, Llor AM, Siqueira HC, Montesinos MJ, Soler LM. Academic training of nursing professionals and its relevance to the workplace. Rev Lat Am Enfermagem. 2015;23(3):404:10.

4. Coelho EBS, et al. Política nacional de atenção integral à saúde do homem. Florianópolis: Universidade Federal de Santa Catarina; 2018. 66 p.

5. LoBiondo-Wood G, Haber J. Desenhos não-experimentais In: LoBiondo-Wood G, Haber J. Pesquisa em enfermagem: métodos, avaliação crítica e utilização. Rio de Janeiro: Guanabara-Koogan; 2011. p. 110-121.

6. Rodrigues VME, Crispim CLM, dos Santos RM, dos Anjos DS. La atención de la salud de la población masculina en los tiempos de la Política Nacional de Atención Integral a la Salud de los hombres: lo que ellos hablan. Cult cuid. 2015; 19(41):135-146.
7. Silveira CLG, Melo VFC, Barreto AJR. Atenção à saúde do homem na atenção primária em saúde: uma revisão integrativa. Rev enferm. UFPE on-line 2017; 11(3): 15281535.

8. Silva GA, Cunha LP, Freire MAM, Tocantins FR. Produção científica, ações profissionais e atenção à saúde do homem: revisão integrativa. Rev enferm. UFPE on line 2016; 10(12):4657-4663.

9. Barreto EF, Crispim JEB, Nogueira WBAG, Almeida SA. A implementação da Política Nacional de Atenção Integral à Saúde do Homem: estado da arte. Rev Pesqui. (Univ. Fed. Estado Rio J., On-line) 2018; 10(Especial):80-84.

10. Aguiar RS, Santana DC, Santana PC. A percepção do enfermeiro da Estratégia Saúde da Família sobre a saúde do homem. R. Enfermagem. Cent. O. Min. 2015; 5(3):18441854 .

11. Alves BMS, Araújo CJS, Almeida SLS, Guimarães ALS. Basic care nurse's activities in connection with difficulties for the implementation of men's health policy. J Nurs UFPE on line 2017; 11(12):5391-5401.

12. Ribeiro DB, Terra MG, Lacchinni AJB, Camponogara S, Beuter M, da Silva CT. Saúde dos homens: abordagem na formação de enfermeiros [Men's health: dealing with training of nurses]. Rev enferm. UERJ 2015; 22(4):540-545.

13. Sousa AP, Silva PES, Dias RS, Azevedo PR, Silva LDC. Avaliação da Política de Atenção Integral à Saúde do Homem. Rev enferm. UFPI 2015; 4(3):65-70.

14. Adamy EK, Lima TL, Teixeira DC, Kutszepa BD, Bernardi GK. Política Nacional de Atenção Integral à Saúde do Homem: visão dos gestores do SUS. Rev Pesqui. (Univ. Fed. Estado Rio J., On-line), 2015; 7(2):2415-2424.

15. Freitas ER, Berardinelli LMM, Espírito Santo FH, Coelho MJ. Nursing care practices in the context of male policy. J Nurs UFPE on-line 2018; 12(2):424-432. 